\title{
Atkins diet program rapidly decreases atherogenic index of plasma in trained adapted overweight men
}

' Departamento de Fisiologia e Biofísica, Instituto de Ciências Biomédicas, Universidade de São Paulo (USP), São Paulo, SP, Brasil

${ }^{2}$ Departamento de Educação Física, Universidade de Mogi das Cruzes (UMC), Mogi das Cruzes, SP, Brasil ${ }^{3}$ Departamento de Educação Física, Faculdade Clube Náutico Mogiano, Mogi das Cruzes, SP, Brasil

* These authors contributed equally to this work

Correspondence to:

Rogério Antonio Laurato Sertié Universidade de São Paulo, Instituto de Ciências Biomédicas Av. Prof. Lineu Prestes, 1524 Ed. Biomédicas I, sala 131 05508-900 - São Paulo, SP, Brasil rsertie@yahoo.com.br

Received on May/12/2015 Accepted on Aug/19/2015

DOI: 10.1590/2359-3997000000106
Rennan de Oliveira Caminhotto ${ }^{{ }^{*}}$, Felipe Lucas Tavares da Fonseca ${ }^{2 \star}$, Natalie Carolina de Castro', João Pedro Arantes ${ }^{3}$, Rogério Antonio Laurato Sertié ${ }^{1}$

\begin{abstract}
Background: The Atkins diet program is a great example of the application of low carbohydrate diets for obesity, with the intention of weight loss and improvement in cardiovascular risk (CV risk). A good CV risk predictor is the atherogenic index of plasma (AIP) calculated as log (TG/HDL [mmol]), which is strongly affected by serum triglycerides, which in turn is associated with the carbohydrate intake. This study determined the effect of the initial phase of Atkins diet program, consisting in 20 g/day of carbohydrate intake with positive urinary ketones measure, in AIP of 12 adult overweight trained adapted men. The AIP was calculated before and after intervention. Results: After 14 days, $\mathrm{BMI}$ and triglycerides decreased significantly, while HDL-C increased. No alterations were described in LDL plasmatic concentration. Prior to the diet, $58.3 \%$ of subjects presented high CV risk and after 14 days of the diet program only $33.3 \%$ of subjects were classified as high CV risk, while more than $66 \%$ were low CV risk. The intervention was effective in 11 of 12 participants. However, in one person the dietary intervention increased AIP index. Conclusion: The initial phase of Atkins diet program could significantly decrease the AIP in 11 of 12 adult overweight trained adapted men. Dietary individual responses need to be more studied. Arch Endocrinol Metab. 2015;59(6):568-71
\end{abstract}

Keywords

Atkins diet; ketosis; cardiovascular risk; overweight; dyslipidemias

\section{INTRODUCTION}

A mong the diets for obesity treatment and metabolic syndrome, a few have gotten a high visibility as low carbohydrate diets, being the Atkins diet program, one of the main examples of this trend. This program is objectively characterized by an unlimited calorie diet, but with limited consumption of carbohydrates about $20 \mathrm{~g} /$ day for at least 14 days and gradual increases, respecting the need for additional weight loss (1).

In addition to the benefits in body weight control, waist circumference, blood pressure and improvement in indicators of carbohydrate metabolism dysfunction, such as fasting plasma glucose, glycated hemoglobin and plasma insulin, months in dietary programs based on carbohydrate restriction has also positively contributed to lipid profile of plasma, increasing HDL cholesterol and decreasing plasma triglycerides (to a great review of these points read) (2).

It's already known that plasma triglycerides is involved in large VLDL particles and small dense LDLs productions, as it has been proposed as the major determinant of cholesterol esterification/transfer and HDL particles remodeling, key determinants in cardiovascular risk, where the smallest LDL and HDL particles have deleterious effects (3). In this context, the plasma parameter $\log$ (TG/HDL-C), well known as 'atherogenic index of plasma' (AIP), has been considered as a marker of plasma atherogenicity, since it indicates the lipoprotein particle size (4) and is associated with coronary artery disease (5).

In this brief communication, we demonstrate that a simple explanation of the initial phase of the Atkins program $(20 \mathrm{~g} / \mathrm{d}$ of carbohydrate during 14 days $)$, even with minimal professional contact, may provide beneficial effects in the AIP in obese and overweight trained adapted individuals.

\section{MATERIALS AND METHODS}

Twelve healthy overweight trained adapted individuals $(\mathrm{n}=12)$ participated voluntarily in this study which was approved by university's institutional ethics committee. The inclusion criteria was based on CV Risk where to participate in the research the voluntary should be rated in high $(>0.21)$ or medium (0.11-0.21). However, 
individuals with low CV risk, but with index above 0.09 (borderline), were included in the research.

To participate in this study the voluntary should be physically adapted. The physical training during the 14 days of diet was muscular resistance exercise, 4 times/ week, in $60 \%$ of maximum intensity previous defined, performing 4 sets of 15 repetitions, with 1 minute interval between them, in order to standardize the training intensity between all participants.

Based in Foster and cols. study (6) the individuals were introduced to the initial phase of Atkins diet program (1) which consists in an unlimited calorie intake of protein and fat, but with a limited carbohydrate intake $(<20 \mathrm{~g} /$ day $)$ for two weeks. The measure of urinary ketones has been done with commercial kit KetoDiabur-Test (Roche) every 3 days. Positive result was needed to proceed the study. Serum analysis was done by a commercial clinical laboratory. AIP was calculated as $\log (\mathrm{TG} / \mathrm{HDL})$ expressed in molar concentrations. Data is expressed in means \pm SEM. Shapiro-Wilk was used to verify normality of the data. Nonparametric paired Student's $t$ test were then used.

\section{RESULTS}

After 14 days in Atkins diet program, body weight, BMI, VLDL and TG, had decreased, while HDL-c increased (Table 1$)$. In addition, the mean $( \pm$ SEM) calculated AIP before the diet program $(0.29 \pm 0.26)$ represented a high CV risk, more precisely in $58,3 \%$ of the individuals (Figure 1B). After 14 days of Atkins diet program, the mean value decreased significantly $(-0.02$ $\pm 0.29, \mathrm{p}<0.05$ Figure 1$)$ and more than $66 \%$ of individuals were low CV risk (Figure $1 \mathrm{~B}$ ).

Table 1. Anthropometric and biochemical data of healthy overweight trained adapted individuals before and after 14 days in Atkins diet program.

\begin{tabular}{lcc}
\hline & Baseline & After diet \\
\hline Age & $30 \pm 1.38$ & - \\
BMl & $31.24 \pm 0.62$ & $28.56 \pm 0.84^{*}$ \\
Body weight (kg) & $98.87 \pm 3.002$ & $93.14 \pm 2.72$ \\
Cholesterol T (mg/dL) & $191.66 \pm 10.61$ & $170 \pm 12.96$ \\
HDL-c (mg/dL) & $32.75 \pm 2.96$ & $38.2 \pm 2.88^{*}$ \\
LDL-c (mg/dL) & $120 \pm 14$ & $112 \pm 16$ \\
VLDL-c (mg/dL) & $20.5 \pm 2.51$ & $12.42 \pm 0.93^{*}$ \\
TG (mg/dL) & $154 \pm 23.73$ & $89.91 \pm 12.44^{*}$ \\
Glycemia (mg/dL) & $86.66 \pm 2.6$ & $88.83 \pm 2.8$ \\
\hline
\end{tabular}

$\mathrm{n}=12 ;{ }^{*} \mathrm{p}<0.05$.

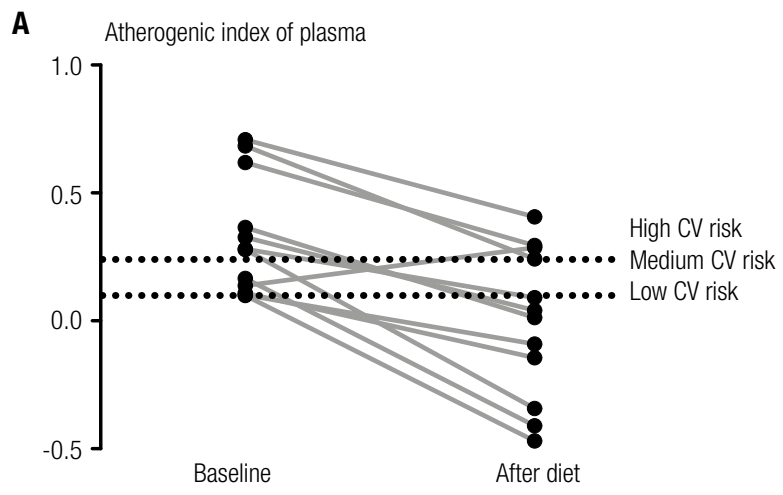

B

\begin{tabular}{ccc}
\hline CV risk & Baseline (\%) & After diet (\%) \\
\hline Low & 25 & 66.7 \\
Medium & 16.7 & 0 \\
High & 58.3 & 33.3 \\
\hline
\end{tabular}

Figure 1. AlP before and after 14 days in Atkins diet program. (A) Each point represents atherogenic indexes of plasma (AIP) of individuals before and after 14 days in initial phase of Atkins diet program. $n=12 ; p<0.05$ (B) Percentage of CV risk before and after diet.

\section{DISCUSSION}

In our study, we have demonstrated that, in addition to body weight loss, only 14 days in Atkins program were enough to provide beneficial effects in HDL-c, TG and, therefore, in AIP. These results are supported by similar studies with more expressive sample size and duration. The recommendation of the Atkins diet program without constant supervision has already been able to promote improvement in the same cardiovascular risk markers after some 2 months to a year (6,7). Although AIP has not been calculated, calculating from the mean of the previously studies cited show similar improvement in AIP values during obesity weight loss. Also, in other study (8), a ketogenic diet with intense carbohydrate restriction has been associated with improvement in serum LDL subclass distribution, increasing the mean of particles diameter after only three weeks of diet, independently of weight loss.

In 2013, a meta-analysis of randomized controlled trials for weight loss (9) demonstrated that carbohydrate restriction promotes a better TG decrease and increase in HDL (the components of AIP index) than conventional restricted-energy high carbohydrate $(>50 \%)$ low fat $(<30 \%)$ diet in more than 1,500 individuals studied for up to a year. Therefore, lack of evidence for high carbohydrate/low fat diets for cardiovascular risk, especially when it comes from serum lipids and cholesterol fraction, and the benefits of fat intake in the same topic 
is part of a constant and current discussion in human nutrition (10). Likewise, despite associations between fat intake and CV risk are commonly cited, large randomized prospective studies never found significant beneficial effects of low fat diets on mortality, cardiovascular disease and risk $(11,12)$ which generates the need of others dietary researches and interventions for blood cholesterol control, particularly LDL and HDL lipoprotein particle size, emphasizing the importance the present results.

A possible explanation of our results is the fact that carbohydrates, especially sugar, interact with plasma lipids due to the changes in hepatic lipid metabolism and short term high carbohydrate diets increases plasma lipids by accelerated VLDL-triglyceride secretion $(13,14)$. Also, once known that low carbohydrate diet is related with lower insulin levels, a shunting in metabolic pathways of hepatic lipogenesis and cholesterol synthesis should be considered. In addition of a higher fat oxidation, syntheses of ketones bodies decrease hepatic substrates of triglycerides and cholesterol (15) which can interfere in serum lipids release and consequently in AIP.

Yet, it should be noticed that a possible interaction between exercise and ketosis might exist, making easier the ketogenic state, even with a higher consumption of protein, that could otherwise stimulate insulin and break ketosis. High protein diets (even high in animal proteins), mainly when carbohydrates are replaced, are not atherogenic and leads to improvement of serum lipid and carbohydrate metabolism, $(16,17)$. Further, it is also discussed that the success of carbohydrate restricted diets depend on the high protein intake (18), leading us not to dismiss the reasonable and possible role of a high protein intake in these results.

Between the usual concerns and misunderstandings about this dietary intervention are the acidosis and kidney healthy. About that: firstly, dietary ketosis (0.5$3.0 \mathrm{mmol}$ ) induced by low carbohydrate intake should not be confused be confused with ketoacidosis (15-25 $\mathrm{mmol})$, a potentially fatal condition which is only possible as a result of uncontrolled diabetes mellitus (19); Secondly, over two years in Atkins diet style, no adverse effects in kidneys were found during obesity treatment with this same approach (20). However, dietary individual responses need to be analyzed. In our results (Figure 1A), we observed a single person who had his AIP index worse after dietary intervention (Medium CV risk to High).
An important limitation of our results is the limited sample size. Nevertheless, our results are supported by current clinical trials and meta-analysis. Therefore, we encourage further research on the topic.

In conclusion, an advice to follow the initial phase of Atkins diet program, which consists in a low-carbohydrate diet with positive urine ketones, can decrease the AIP after only 14 days of program, in adult obese or overweight trained adapted men. This finding may reflect positively in clinical nutrition.

Author contributions: Rennan de Oliveira Caminhotto and Felipe Lucas Tavares da Fonseca: design and conduct of the study, data analysis and manuscript preparation. Rennan de Oliveira Caminhotto and Rogério Antonio Laurato Sertié: manuscript writing. Rennan de Oliveira Caminhotto, Rogério Antonio Laurato Sertié, Natalie Carolina de Castro and João Pedro Arantes: data interpretation. Rogério Antonio Laurato Sertié: coordination of research.

Funding source: scientific initiation scholarships by $\mathrm{CNPq}(\mathrm{Na}-$ tional Council for Scientific and Technological Development) for Felipe Lucas Tavares da Fonseca. Post Doctoral scholarships by FAPESP (13/13601-2) for Rogério Antonio Laurato Sertié and Doctoral scholarships for Rennan de Oliveira Caminhotto (14/10705-4).

Acknowledgements: we thank Bruno Simonetti for initial English review.

Disclosure: no potential conflict of interest relevant to this article was reported.

\section{REFERENCES}

1. Atkins RC. Dr. Atkins New Diet Revolution (Revised Edition). Lanham, MD: M. Evans \& Company; 2002.

2. Santos FL, Esteves SS, da Costa Pereira A, Yancy WS Jr, Nunes JP. Systematic review and meta-analysis of clinical trials of the effects of low carbohydrate diets on cardiovascular risk factors. Obes Rev. 2012;13:1048-66.

3. Dobiásová M, Frohlich J, Sedová M, Cheung MC, Brown BG. Cholesterol esterification and atherogenic index of plasma correlate with lipoprotein size and findings on coronary angiography. $J$ Lipid Res. 2011;52:566-71.

4. Dobiásová $\mathrm{M}$, Frohlich J. The plasma parameter log (TG/HDL-C) as an atherogenic index: correlation with lipoprotein particle size and esterification rate in apoB-lipoprotein-depleted plasma (FER(HDL)). Clin Biochem. 2001;34:583-8.

5. Frohlich J, Dobiásová M. Fractional esterification rate of cholesterol and ratio of triglycerides to HDL-cholesterol are powerful predictors of positive findings on coronary angiography. Clin Chem. 2003;49:1873-80.

6. Foster GD, Wyatt HR, Hill JO, McGuckin BG, Brill C, Mohammed $\mathrm{BS}$, et al. A randomized trial of a low-carbohydrate diet for obesity. N Engl J Med. 2003;348:2082-90.

7. Gardner CD, Kiazand A, Alhassan S, Kim S, Stafford RS, Balise $\mathrm{RR}$, et al. Comparison of the Atkins, Zone, Ornish, and LEARN 
diets for change in weight and related risk factors among overweight premenopausal women: the ATO Z Weight Loss Study: a randomized trial. JAMA. 2007;297:969-77.

8. Sharman MJ, Kraemer WJ, Love DM, Avery NG, Gómez AL, Scheett TP, et al. A ketogenic diet favorably affects serum biomarkers for cardiovascular disease in normal-weight men. $J$ Nutr. 2002;132:1879-85.

9. Bueno NB, de Melo IS, de Oliveira SL, da Rocha Ataide T. Verylow-carbohydrate ketogenic diet v. low-fat diet for long-term weight loss: a meta-analysis of randomised controlled trials. $\mathrm{Br}$ J Nutr. 2013;110:1178-87.

10. DiNicolantonio JJ. The cardiometabolic consequences of replacing saturated fats with carbohydrates or $\Omega-6$ polyunsaturated fats: Do the dietary guidelines have it wrong? Open Heart. 2014; $1: \mathrm{e} 000032$

11. Multiple risk factor intervention trial. Risk factor changes and mortality results. Multiple Risk Factor Intervention Trial Research Group. JAMA. 1982;248:1465-77.

12. Howard BV, Van Horn L, Hsia J, Manson JE, Stefanick ML, Wassertheil-Smoller $\mathrm{S}$, et al. Low-fat dietary pattern and risk of cardiovascular disease: the Women's Health Initiative Randomized Controlled Dietary Modification Trial. JAMA. 2006;295:655-66.
13. Mittendorfer B, Sidossis LS. Mechanism for the increase in plasma triacylglycerol concentrations after consumption of shortterm, high-carbohydrate diets. Am J Clin Nutr. 2001;73:892-99.

14. Jeppesen J, Chen YD, Zhou MY, Wang T, Reaven GM. Effect of variations in oral fat and carbohydrate load on postprandial lipemia. Am J Clin Nutr. 1995;62:1201-5.

15. Westman EC, Mavropoulos J, Yancy WS, Volek JS. A review of low carbohydrate ketogenic diets. Curr Atheroscler Rep. 2003;5:476-83.

16. Skov AR, Toubro S, Rønn B, Holm L, Astrup A. Randomized trial on protein vs carbohydrate in ad libitum fat reduced diet for the treatment of obesity. Int J Obes Relat Metab Disord. 1999;23:528-36.

17. Cordain L, Eaton SB, Miller JB, Mann N, Hill K. The paradoxical nature of hunter-gatherer diets: meat-based, yet non-atherogenic. Eur J Clin Nutr. 2002;56(Suppl 1):S42-52.

18. Soenen S, Bonomi AG, Lemmens SG, Scholte J, Thijssen MA, van Berkum $F$, et al. Relatively high-protein or 'low-carb' energyrestricted diets for body weight loss and body weight maintenance? Physiol Behav. 2012;107:374-80.

19. Veech RL, Chance B, Kashiwaya Y, Lardy HA, Cahill GF Jr. Ketone bodies, potential therapeutic uses. IUBMB Life. 2001;51:241-7.

20. Friedman AN, Ogden LG, Foster GD, Klein S, Stein R, Miller B, et al. Comparative effects of low-carbohydrate high-protein versus low-fat diets on the kidney. Clin J Am Soc Nephrol. 2012;7:1103-11. 